Results Maps show the temporal evolution and spatial distribution of dengue fever risk on the territory. Highest risk areas coincide with those of greater movement of people and lack of infrastructure in the municipality.

Conclusion The method was suitable for identifying risk areas, in which government's actions should be efficient and constant.

\section{P1-342 EMPIRICAL ANALYSIS OF THE RISK ESTIMATORS IN SPATIAL CASE-CONTROL STUDIES USING DIFFERENT SAMPLE DESIGNS}

doi:10.1136/jech.2011.142976f.34

${ }^{1} \mathrm{~A}$ C C N Mafra, ${ }^{2} \mathrm{C}$ T Codeco, ${ }^{1} \mathrm{R}$ Cordeiro, ${ }^{1} \mathrm{C}$ Stephan, ${ }^{* 1} \mathrm{~L}$ B Nucci. ${ }^{1}$ State University of Campinas, Campinas, São Paulo, Brazil; 'Fundação Oswaldo Cruz, Rio de Janeiro, Brazil

Introduction There are many sample designs for case-control studies. Three of them were simulated to investigate the properties of their risk estimators when the aim of the study is to analyse the space along with other covariates. They are: the case-base sampling, where all controls are selected at the beginning while the cases are sampled during the study as they occurs; the survivor sampling, in which both cases and controls are sampled at the end of the study; and the risk-set sampling, where both cases and controls are sampled during the study.

Methods A realistic at risk population was created by sampling individuals from the empirical spatial distributions derived from governmental census information of a Brazilian city. Two epidemic scenarios were built, a transmissible and a nontransmissible disease. We used the generalised additive models to estimate the risks in each different study, fitting semiparametric models with the geographical coordinates and other covariates as age, income, gender and study.

Results The results suggest that the estimated spatial risks are similar in the three sample designs, but the standard deviations vary in the space and, the widest variation occurs in the survivor sampling (for the nontransmissible disease) and in the case-base sampling (for the transmissible disease). The parametric estimates that are closest to the initially defined were attained by the risk-set sampling, at the nontransmissible disease scheme.

Conclusion We conclude that the best risk estimates are attained by sampling the controls at the same time of the cases, as the epidemic occurs.

\section{P1-343 GENDER DIFFERENCES IN HOME SMOKING RESTRICTIONS IN HUNGARY}

doi:10.1136/jech.2011.142976f.35

${ }^{1} \mathrm{~L}$ Nagymajtényi, ${ }^{1}{ }^{1} \mathrm{~A}$ Maróti-Nagy, ${ }^{2} \mathrm{D}$ Easterling, ${ }^{3} \mathrm{~T}$ Rogers, ${ }^{1} \mathrm{E}$ Paulik. ${ }^{1}$ Department of Public Health, Faculty of Medicine, University of Szeged, Szeged, Hungary; ${ }^{2}$ Wake Forest University School of Medicine, Winston-Salem, USA; ${ }^{3}$ Public Health Institute, Oakland, USA

This publication was made possible by Grant Number 1 R01 TW007927-01 from the Fogarty International Center, the National Cancer Institute, and the National Institutes on Drug Abuse, within the National Institutes of Health (NIH). Its contents are solely the responsibility of the authors and do not necessarily represent the official view of the NIH.

Introduction The relationship between the environmental tobacco smoke exposure and the increased risk of illness and death from a wide range of diseases is well-known. The aim of this study was to assess the connection between gender and home smoking restrictions.

Methods The first wave of a quantitative longitudinal study was delivered in 2009. A sample of individuals $(n=2250)$ aged
16-70 years completed a self-administered questionnaire (response rate $=57.4 \%$ ). Logistic regression models were applied to reveal the predictors of having a smoke-free home in smokers and non-smokers. Results Nearly two-third of people $34.9 \%$ of smokers and $71.4 \%$ of non-smokers) lived in homes where smoking was totally restricted. The predictors of having a smoke-free home were gender (female: $\mathrm{OR}=1.60 ; \mathrm{p}<0.0001$ ) and education (medium: $\mathrm{OR}=1.73$; high: $\mathrm{OR}=1.96 ; \mathrm{p}<0.001$ ) among non-smokers; while education (medium: $\mathrm{OR}=1.81$; high: $\mathrm{OR}=1.3 .20 ; \mathrm{p}<0.001)$ and living in the rural area $(\mathrm{OR}=1.91 ; \mathrm{p}<0.0001)$ in smokers.

Conclusion Home smoking restrictions were associated with gender and education among non-smokers, with education and living place among smokers. The results showed that greater attention must be placed on non-smoker males, on smokers living in urban areas, and in general on low educated people to improve tobacco control in Hungary.

\section{P1-344 CORONARY ARTERY LESIONS OF INCOMPLETE KAWASAKI DISEASE: THE NATIONWIDE SURVEY OF 2007-2008 IN JAPAN}

doi:10.1136/jech.2011.142976f.36

${ }^{1} \mathrm{D}$ Sudo, ${ }^{*} \mathrm{Y}$ Monobe, ${ }^{1} \mathrm{M}$ Yashiro, ${ }^{1} \mathrm{M}$ Mieno, ${ }^{1} \mathrm{R}$ Uehara, ${ }^{2} \mathrm{~K}$ Tsuchiya, ${ }^{2} \mathrm{~T}$ Sonobe, ${ }^{1} Y$ Nakamura. ${ }^{1}$ Jichi Medical University, Shimotsuke, Japan; ${ }^{2}$ Japanese Red Cross Medical Center, Shibuya-ku, Japan

Objectives To observe the recent epidemiologic features of incomplete Kawasaki disease (KD) patients reported to the Japanese nationwide survey, and to compare risk factors for developing coronary artery lesions (CALs) between incomplete $\mathrm{KD}$ and complete $\mathrm{KD}$.

Methods In the 2007-2008 nationwide survey, $23337 \mathrm{KD}$ patients were reported and then classified by their number of principal symptoms for analyses. In this study, complete $\mathrm{KD}$ was defined as having five or more of the six principal symptoms; incomplete $\mathrm{KD}$ as having four or fewer regardless of the presence or absence of CALs.

Results Compared with complete KD patients, younger ages at first hospital visit, a higher incidence rate of CALs, less frequency of both initial and additional intravenous immunoglobulin (IVIG) administration, later IVIG administration, less total dose of IVIG administration, and less frequency of steroid therapy were observed among those with incomplete $\mathrm{KD}$. Also particularly in patients having CALs, the same delayed and insufficient IVIG therapy was observed in incomplete $\mathrm{KD}$. Multivariate analysis showed that the risk factors for CALs observed in incomplete $\mathrm{KD}$ were almost the same as those observed in complete $\mathrm{KD}$, except for receiving initial IVIG therapy. The initial IVIG administration was risk of developing CALs in incomplete $\mathrm{KD}$, but not risk rather protective in complete $\mathrm{KD}$.

Conclusions The results imply the existence of a common pathological basis in generating CALs regardless of presentation types of $\mathrm{KD}$. Therefore, more timely diagnosis and treatment to incomplete $\mathrm{KD}$ patients could lead to the further prevention of cardiac lesions caused by $\mathrm{KD}$

\section{P1-345 REGULATORY RISK ASSESSMENT IN EPIDEMIOLOGY: PRINCIPLES AND METHODOLOGY}

doi:10.1136/jech.2011.142976f.37

Y Sun, ${ }^{*}$ F Bochmann. Institute for Occupational Safety and Health of German Social Accident Insurance (IFA), Sankt Augustin, Germany

Regulatory risk assessment is a useful tool in the development of public policy, regulation and decision making in occupational safety and health. It focuses on a detailed quantification of occupational 